MATHEMATICS OF COMPUTATION

S 0025-5718(99)01158-8

Article electronically published on August 18, 1999

\title{
A POSTERIORI ERROR ESTIMATES FOR UPWIND FINITE VOLUME SCHEMES FOR NONLINEAR CONSERVATION LAWS IN MULTI DIMENSIONS
}

\author{
DIETMAR KRÖNER AND MARIO OHLBERGER
}

\begin{abstract}
In this paper we shall derive a posteriori error estimates in the $L^{1}$-norm for upwind finite volume schemes for the discretization of nonlinear conservation laws on unstructured grids in multi dimensions. This result is mainly based on some fundamental a priori error estimates published in a recent paper by $\mathrm{C}$. Chainais-Hillairet. The theoretical results are confirmed by numerical experiments.
\end{abstract}

\section{INTRODUCTION}

One of the most important tools for accelerating comprehensive computations in multi dimensions (in particular in 3-D) is the local adaption of the grid. In order to minimize the computing time the local grid size should be chosen such that the error $\left\|u-u_{h}\right\|$ between the exact solution $u$ and the numerical solution $u_{h}$ is less than a given tolerance and such that the total number of cells is as small as possible. This can be obtained if an a posteriori error estimate of the form

$$
\left\|u-u_{h}\right\| \leq c \sum_{j} \eta_{j}\left(h_{j}\right)+R_{h}
$$

is given, where $\eta_{j}\left(h_{j}\right)$ are local quantities related to the diameter $h_{j}$ of each cell or edge such that $\eta_{j}\left(h_{j}\right)$ can be computed if $u_{h}$ is known, and where $R_{h}$ is related to the approximation of the data, with $h:=\max _{j} h_{j}$. Also the constant $c$ should be known. If $\eta_{j}\left(h_{j}\right)$ and $R_{h}$ become sufficiently small when $h_{j}$ and $h$ tend to zero, the right hand side in (1) can be made small by local mesh refinement.

For elliptic and parabolic problems the theory of a posteriori error estimates of the form (1) is well developed [6], [7], [8], [9], [19]. But up to now there are no analogous results for initial value problems for nonlinear conservation laws of the form

$$
\begin{gathered}
\partial_{t} u+\operatorname{div} F(x, t, u)=0 \quad \text { in } \mathbb{R}^{N} \times \mathbb{R}^{+}, \\
u(x, 0)=u_{0}(x) \text { in } \mathbb{R}^{N} .
\end{gathered}
$$

Received by the editor February 5, 1998.

1991 Mathematics Subject Classification. Primary 65M15, 35L65, 76M25.

Key words and phrases. A posteriori error estimates, conservation laws, finite volume schemes, adaptive methods, unstructured grids. 
Instead of rigorous error estimators, in many applications error indicators, shock indicators or grid indicators have been used in order to find those regions with steep gradients. Usually these indicators are based on discrete gradients or higher order discrete derivatives of the discrete solution. They are used to control the local process of refining and coarsening the grid. But these indicators give no information about the true error $\left\|u-u_{h}\right\|$.

In this paper we shall exhibit a rigorous a posteriori error estimate of the form (1) in the $L^{1}$-norm in space and time. Earlier results related to this topic were published in [2], [3], [4], [5], [10], [14], [15], [17], [18], [20] for the nonlinear case and in [9], [13], [14], [16], [19] for linear systems of equations. In Section 2 we will fix the notation and present the main result. Furthermore we will quote the fundamental estimates from [1] and put them together in order to derive the main result. Numerical examples will be given in Section 3.

\section{Notation AND MAin RESUlt}

In this section we will fix the notations and assumptions, define the upwind finite volume scheme for solving (2) and (3), and present the a posteriori estimate, the main result of this paper. For the data we have to assume the following conditions:

$$
\begin{gathered}
u_{0} \in L^{\infty}\left(\mathbb{R}^{N}\right) \cap B V_{l o c}\left(\mathbb{R}^{N}\right) \quad \text { with } A, B \in \mathbb{R} \text { such that } A \leq u_{0} \leq B \quad \text { a.e. } \\
F \in C^{1}\left(\mathbb{R}^{N} \times \mathbb{R}^{+} \times \mathbb{R}, \mathbb{R}^{N}\right), \\
\sum_{j} \frac{\partial F_{j}}{\partial x_{j}}(x, t, s)=0 \quad \text { for all }(x, t, s) \in \mathbb{R}^{N} \times \mathbb{R}^{+} \times \mathbb{R} .
\end{gathered}
$$

For all compact sets $K \subset \mathbb{R}$ there exists a constant $c_{0}(K)$ such that

$$
\begin{aligned}
\left|\partial_{s} F(x, t, s)\right| & \leq c_{0}(K), \\
\left|\partial_{x} \partial_{s}^{\beta} F(x, t, s)\right|+\left|\partial_{t} \partial_{s}^{\beta} F(x, t, s)\right| & \leq c_{0}(K)
\end{aligned}
$$

for almost all $(x, t, s) \in \mathbb{R}^{N} \times \mathbb{R}^{+} \times K$ and $\beta \in\{0,1\}$.

Remark 2.1. Under the above assumptions above the existence of an entropy solution of (2) and (3) is proved in Theorem 5 of [1]. The uniqueness follows from Theorem 6 of [1].

For $t_{n} \in \mathbb{R}^{+}$let $\mathcal{T}^{n}=\left\{T_{j} \mid j \in I^{n}\right\}$ be a mesh of $\mathbb{R}^{N}$ such that the interface of two neighbouring cells $T_{j}, T_{l}$ of $\mathcal{T}^{n}$ is included in a hyperplane (see also [1]). The joint edge of $T_{j}$ and $T_{l}$ will be denoted by $S_{j l}$. Let $h_{j}:=\operatorname{diam} T_{j}$ and $h_{\min }^{n}:=\min _{j \in I^{n}} h_{j}$. We assume that $h_{\min }^{n}>0$ and that there exists an $\alpha>0$ such that for all $h_{j}>0$ we have

$$
\begin{aligned}
\alpha h_{j}^{N} & \leq \operatorname{meas}\left(T_{j}\right), \\
\alpha \text { meas }\left(S_{j l}\right) & \leq h_{j}^{N-1}
\end{aligned}
$$

for all $n$ and for all $j, l \in I^{n}$. Define $h_{j l}:=\max \left\{\operatorname{diam} T_{j}\right.$, $\left.\operatorname{diam} T_{l}\right\}$, which gives us the estimate $\left|S_{j l}\right| \leq h_{j l}^{N-1}$. Of course $T_{j}, S_{j l}, h_{j}$ and $h_{j l}$ depend on $n$, but for simplicity we suppress the index $n$ here.

Remark 2.2. In the situation where $\Delta t^{n}=t^{n+1}-t^{n}$ and the $\mathcal{T}^{n}$ are constant in time and $h_{j}$ can be replaced by $h:=\max _{j} \operatorname{diam}\left(T_{j}\right)$, the condition (9) corresponds 
exactly to condition (5) in [1]. Thus all results of [1] hold in this more restrictive situation.

Nevertheless we have verified that all results of [1] used in this article to prove the a posteriori error estimates hold true in the more general situation (9).

For any $j, l \in I^{n}$ and $t_{n} \in \mathbb{R}^{+}$there is a numerical flux $g_{j l}^{n}: \mathbb{R}^{2} \rightarrow \mathbb{R}$ which satisfies the following conditions for all $u, v, u^{\prime}, v^{\prime} \in[A, B]$ :

The numerical flux $g_{j l}^{n}(u, v)$ is monotone increasing with respect to $u$ and monotone decreasing with respect to $v$.

Furthermore there exists a constant $L$, independent of $j, l, n$ and $h$, such that for all $u, v, u^{\prime}, v^{\prime}$ as above

$$
\begin{gathered}
g_{j l}^{n}(u, v)=-g_{l j}^{n}(v, u) \\
\left|g_{j l}^{n}(u, v)-g_{j l}^{n}\left(u^{\prime}, v^{\prime}\right)\right| \leq L\left|S_{j l}\right|\left(\left|u-u^{\prime}\right|+\left|v-v^{\prime}\right|\right)
\end{gathered}
$$

and

$$
g_{j l}^{n}(u, u)=\frac{1}{\Delta t^{n}} \int_{t^{n}}^{t^{n+1}} \int_{S_{j l}} F(x, t, u) n_{j l} d x d t
$$

where $\Delta t^{n}$ is the time step, $t^{n}:=\sum_{l=1}^{n} \Delta t^{l}$ and $n_{j l}$ is the outer unit normal to $S_{j l}$ with respect to $T_{j}$.

From the assumption (8), (13) we obtain the following fact.

Lemma 2.3. There exists a constant $M_{1}$ such that

$$
\left|F(x, t, v) n_{j l}-\frac{1}{\left|S_{j l}\right|} g_{j l}^{n}(v, v)\right| \leq M_{1}\left(h_{j l}+\Delta t^{n}\right) .
$$

Proof. It follows from the assumptions (8), (13).

Remark 2.4. If $F(x, t, v)=F(v)$, then the condition (13) implies that $M_{1}=0$ and that the left-hand side in (14) is equal to zero.

Now the upwind finite volume scheme for computing approximate solutions to (2), (3) is defined by

Definition 2.5 (Finite volume scheme). Let

$$
\begin{aligned}
u_{j}^{0} & :=\frac{1}{\left|T_{j}\right|} \int_{T_{j}} u_{0}, \\
u_{j}^{n+1} & :=u_{j}^{n}-\frac{\Delta t^{n}}{\left|T_{j}\right|} \sum_{l \in N(j)} g_{j l}^{n}\left(u_{j}^{n}, u_{l}^{n}\right)
\end{aligned}
$$

for all $n \in \mathbb{N}$ and $j, l \in I^{n}$. Here $N(j)$ denotes the indices of the neighbouring cells of $T_{j}$.

For the time step $\Delta t^{n}$ we assume the following CFL-condition for given $\left.\xi \in\right] 0,1[$ :

$$
\Delta t^{n} \leq \frac{(1-\xi) \alpha^{2} h_{\min }^{n}}{2 L}
$$

where $L$ is the Lipschitz constant from (12). Let us denote

$$
u_{h}(x, t):=u_{j}^{n} \quad \text { if } \quad x \in T_{j}\left(=T_{j}^{n}\right), \quad t^{n}<t \leq t^{n+1} .
$$


Lemma 2.6 ( $\mathbf{L}^{\infty}$ stability). Assume that the assumptions (4),..,(13), (16) are fulfilled. Then the approximate solution $u_{h}$ defined in (17) satisfies

$$
A \leq u_{j}^{n} \leq B, \forall n \in \mathbb{N}, \forall j \in I^{n},
$$

and

$$
\left\|u_{h}\left(t^{n}\right)\right\|_{L^{\infty}\left(\mathbb{R}^{N}\right)} \leq\left\|u_{0}\right\|_{L^{\infty}\left(\mathbb{R}^{N}\right)}, \forall n \in \mathbb{N} .
$$

Proof. Repeat the proof of Lemma 1 in [1], where the global CFL-condition is replaced by the local conditions (9) and (16).

Let $u$ be the exact solution of (2), (3) and $u_{h}$ be the discrete solution as defined in (17). In [1], [2], [20], it was shown that in the uniform case (i.e. $h_{j}$ in $(9)$ can be replaced by $\left.h:=\max _{j} \operatorname{diam}\left(T_{j}\right)\right)$ under the assumptions $(4), \ldots,(13),(16)$ we have for any compact set $K \subset \mathbb{R}^{N} \times \mathbb{R}^{+}$

$$
\int_{K}\left|u(x, t)-u_{h}(x, t)\right| d x d t \leq \operatorname{ch}^{\frac{1}{4}}
$$

where the constant c depends only on $K$ and the data $F, u_{0}, L, c_{0}, \alpha$ and $\xi$.

Now it turns out that the same tools which have been used for proving (18) in [1] can be used to show an a posteriori estimate. In order to present the details we have to define the following measures as in [1].

Definition 2.7. (Measures) For $\phi \in C_{0}^{0}\left(\mathbb{R}^{N}\right), \phi \in C_{0}^{0}\left(\mathbb{R}^{N} \times \mathbb{R}^{+}\right)$respectively let

$$
\begin{aligned}
\left\langle\mu_{h}^{0}, \phi\right\rangle:= & \int_{\mathbb{R}^{N}}\left|u_{0}(x)-u_{h}(x, 0)\right| \phi(x) d x \\
\left\langle\lambda_{h}, \phi\right\rangle:= & \sum_{n} \sum_{j}\left|u_{j}^{n+1}-u_{j}^{n}\right| \int_{t^{n}}^{t^{n+1}} \int_{T_{j}} \phi(x, t) d x d t, \\
\left\langle\mu_{j l}^{n}, \phi\right\rangle:= & \frac{1}{\left(\Delta t^{n}\right)^{2}\left|T_{j}\right|\left|S_{j l}\right|} \int_{t^{n}}^{t^{n+1}} \int_{T_{j}} \int_{t^{n}}^{t^{n+1}} \int_{S_{j l}} \int_{0}^{1}\left(h_{j l}+\Delta t^{n}\right) \\
\left\langle\nu_{j l}^{n}, \phi\right\rangle:= & \frac{1}{\Delta t^{n}\left|S_{j l}\right|} \int_{t^{n}}^{t^{n+1}} \int_{S_{j l}} \int_{t^{n}}^{t^{n+1}} \int_{S_{j l}} \int_{0}^{1}\left(h_{j l}+\Delta t^{n}\right)^{2} \phi(\xi+\theta(\gamma-\xi), \\
\left\langle\mu_{h}, \phi\right\rangle:= & \left\langle\lambda_{h}, \phi\right\rangle+2 \sum_{n} \sum_{\text {edges }} \Delta t^{n}\left(S_{j l}^{1}+S_{j l}^{2}\right) \\
& +2 M_{1} \sum_{n} \sum_{\text {edges }}\left\langle\nu_{j l}^{n}, \phi\right\rangle,
\end{aligned}
$$

where $n \in \mathbb{N}, \sum_{\text {edges }}$ refers to the sum over all edges of the mesh with neighbouring triangles $T_{j}, T_{l}$ and

$$
\begin{aligned}
S_{j l}^{1} & =\max _{u_{l}^{n} \leq c \leq d \leq u_{j}^{n}}\left(g_{j l}^{n}(d, c)-g_{j l}^{n}(d, d)\right)\left\langle\mu_{j l}^{n}, \phi\right\rangle, \\
S_{j l}^{2} & =\max _{u_{l}^{n} \leq c \leq d \leq u_{j}^{n}}\left(g_{j l}^{n}(d, c)-g_{j l}^{n}(c, c)\right)\left\langle\mu_{j l}^{n}, \phi\right\rangle .
\end{aligned}
$$

Lemma 2.8. Assume (4),..,(13), (16). Let $u$ and $u_{h}$ be the exact solution of (2) and the discrete solution of (15) respectively. Then for all $R>0, T>0$ there exist 
constants $M_{2}, M_{3}>0$ such that in the case of a uniform mesh (i.e. $h_{j}$ in (9) can be replaced by $h:=\max _{j} \operatorname{diam}\left(T_{j}\right)$ )

$$
\begin{aligned}
\mu_{h}\left(B_{R}(0) \times[0, T]\right) & \leq M_{2}(h+\sqrt{h}), \\
\mu_{h}^{0}\left(B_{R}(0)\right) & \leq M_{3} h
\end{aligned}
$$

uniformly in $h$.

Proof. See [1], Theorem 4.

Also, from Theorem 4 in [1] we obtain

Theorem 2.9. Assume (4),...,(13), (16). Let $u$ and $u_{h}$ be the exact solution of (2) and the discrete solution of (15) respectively. Let $\mu_{h}, \mu_{h}^{0}$ be defined as in Definition 2.7. Then for all $k \in \mathbb{R}$ and $\phi \in C_{0}^{\infty}\left(\mathbb{R}^{N} \times \mathbb{R}^{+}, \mathbb{R}^{+}\right)$we have

$$
\begin{gathered}
\int_{\mathbb{R}^{N} \times \mathbb{R}^{+}}\left|u_{h}-k\right| \partial_{t} \phi+\left(F\left(x, t, \max \left\{u_{h}, k\right\}\right)-F\left(x, t, \min \left\{u_{h}, k\right\}\right)\right) \nabla \phi d x d t \\
+\int_{\mathbb{R}^{N}}\left|u_{0}-k\right| \phi(x, 0) d x \\
\geq-\int_{\mathbb{R}^{N} \times \mathbb{R}^{+}}\left(\left|\partial_{t} \phi\right|+|\nabla \phi|\right) d \mu_{h}-\int_{\mathbb{R}^{N}} \phi(x, 0) d \mu_{h}^{0} .
\end{gathered}
$$

Proof. This follows by integration by parts and using the definition of $u_{h}$ in (17) and Definition 2.5 (cf. [1], Theorem 4). Note that this part of the proof of Theorem 4 in [1] also holds in the general case of nonuniform grids as described in (9).

For the following lemma we need

$$
\mathcal{M}(\Omega):=\text { the set of positive continuous linear forms on } C^{0}(\Omega) .
$$

Lemma 2.10. Assume (4),..,(7), $u_{0} \in B V\left(\mathbb{R}^{N}\right), \tilde{u} \in L^{\infty}\left(\mathbb{R}^{N} \times \mathbb{R}^{+}\right), A \leq \tilde{u} \leq$ $B$ a.e., that there exist measures $\mu \in \mathcal{M}\left(\mathbb{R}^{N} \times \mathbb{R}^{+}\right), \mu^{0} \in \mathcal{M}\left(\mathbb{R}^{N}\right)$ such that the statement of Theorem 2.9 holds with $\tilde{u}$ instead of $u_{h}$, and that $u$ is the unique solution of (2), (3).

Let $\omega \in \mathbb{R}^{+}$be given, and let $\rho \in C_{0}^{1}\left(\mathbb{R}^{+} ;[0,1]\right)$ be such that $\rho^{\prime} \leq 0$ and

$$
\begin{array}{lll}
\rho=1 & \text { on } & {[0, R],} \\
\rho=0 & \text { on } & {[R+1, \infty]}
\end{array}
$$

and

$$
\begin{aligned}
& \psi(x, t):=\frac{T-t}{T} \rho\left(\left|x-x_{0}\right|+\omega t\right) \quad \text { on } \quad \mathbb{R}^{N} \times[0, T], \\
& \psi(x, t):=0 \quad \text { on } \quad \mathbb{R}^{N} \times[T, \infty] .
\end{aligned}
$$

Then we have

$$
\begin{gathered}
\int_{\mathbb{R}^{N} \times \mathbb{R}^{+}}|\tilde{u}-u| \partial_{t} \psi+(F(x, t, \max \{\tilde{u}, u\})-F(x, t, \min \{\tilde{u}, u\})) \nabla \psi d x d t \\
\geq-a_{0} \mu^{0}(\{\psi(., 0) \neq 0\})-2 \sqrt{b c} \mu(\{\psi \neq 0\})^{\frac{1}{2}}-a \mu(\{\psi \neq 0\}),
\end{gathered}
$$


with

$$
\begin{aligned}
a:= & 2 \omega+\frac{1}{T}+2, \\
a_{0}:= & 1 \\
b:= & 4+2^{N+2} \\
c:= & \|u\|_{B V}\left(2\left(2 \omega+\frac{1}{T}\right)+\left(8+2^{N+5}\right) c_{0}([A, B])\right) \\
& +\left\|u_{0}\right\|_{B V}\left(2^{N+4} c_{0}([A, B])+1\right) \\
& +2 c_{0}([A, B]) \max \{|A|,|B|\}\left(\left|B_{R+1}(0)\right|-\left|B_{R}(0)\right|\right) T,
\end{aligned}
$$

where $c_{0}$ is the constant in (7) and (8).

Proof. See [1], Lemma 8. To get the constants $a, a_{0}, b$ and $c$ one has to repeat the arguments in [1] and control all constants which appear in the estimates.

Now we can present the a posteriori error estimate, the main result of this paper. Let $R, \omega=c_{0}([A, B])$ (see $\left.(7)\right)$, and $T$ be given, and let

$$
\begin{aligned}
I_{0}: & =\left\{n \mid 0 \leq t^{n} \leq \min \left\{\frac{R+1}{\omega}, T\right\}\right. \\
D_{R+1}: & =\left\{(x, t)|| x-x_{0} \mid+\omega t<R+1\right\} \\
M(t): & =\left\{j \mid \text { there exists } x \in T_{j} \text { such that }(x, t) \in D_{R+1}\right\} .
\end{aligned}
$$

Theorem 2.11. Assume the conditions are as in Theorem 2.9 and let $u_{0} \in$ $B V\left(\mathbb{R}^{N}\right)$. Let $K \subset \subset \mathbb{R}^{N} \times \mathbb{R}^{+}, \omega=c_{0}([A, B]$ ) (see (7)) and choose $T, R$ and $x_{0}$ such that $\left.T \in\right] 0, \frac{R}{\omega}[$ and

$$
K \subset \bigcup_{0 \leq t \leq T} B_{R-\omega t}\left(x_{0}\right) \times\{t\} .
$$

Then we have

$$
\int_{K}\left|u-u_{h}\right| \leq T\left[a_{0} \int_{\left|x-x_{0}\right|<R+1}\left|u_{0}(x)-u_{h}(x, 0)\right| d x+a Q+2 \sqrt{b c Q}\right],
$$

where

$$
\begin{aligned}
Q: & =\sum_{n \in I_{0}} \sum_{j \in M\left(t^{n}\right)}\left|u_{j}^{n+1}-u_{j}^{n}\right| \Delta t^{n} h_{j}^{N} \\
& +2 \sum_{n} \sum_{\text {edges }} \Delta t^{n}\left(\Delta t^{n}+h_{j l}\right) \max _{u_{l}^{n} \leq c \leq d \leq u_{j}^{n}}\left(g_{j l}^{n}(d, c)-g_{j l}^{n}(d, d)\right) \delta_{j l}^{n} \\
& +2 \sum_{n} \sum_{\text {edges }} \Delta t^{n}\left(\Delta t^{n}+h_{j l}\right) \max _{u_{l}^{n} \leq c \leq d \leq u_{j}^{n}}\left(g_{j l}^{n}(d, c)-g_{j l}^{n}(c, c)\right) \delta_{j l}^{n} \\
& +2 M_{1} \sum_{n} \sum_{\text {edges }} \Delta t^{n} h_{j l}^{N-1}\left(\Delta t^{n}+h_{j l}\right)^{2} \delta_{j l}^{n}
\end{aligned}
$$

and

$$
\begin{aligned}
& \delta_{j l}^{n}:=0 \quad \text { if }\left(T_{j} \cup T_{l}\right) \times\left[t^{n}, t^{n+1}\right] \cap D_{R+1}=\emptyset \\
& \delta_{j l}^{n}:=1 \text { otherwise. }
\end{aligned}
$$




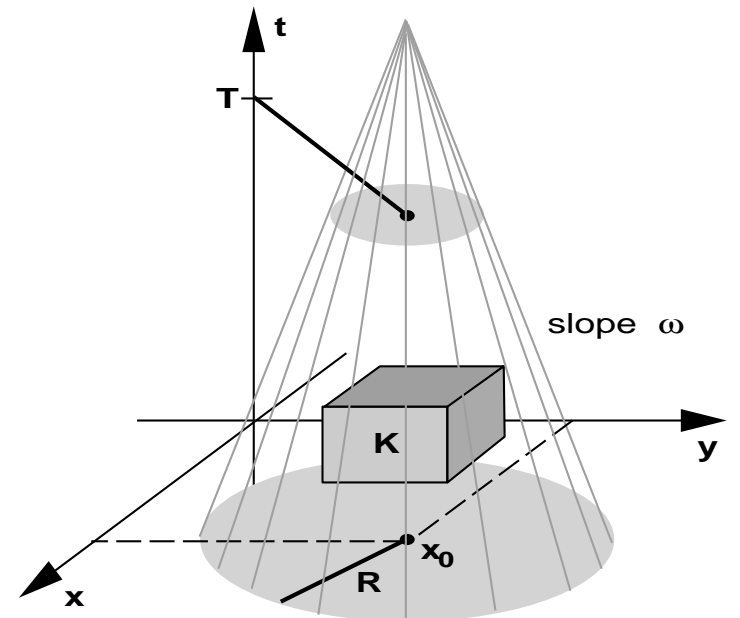

Figure 1. The relation between $\omega, R, T$ and $K$.

Proof. By assumption we have

$$
K \subset \bigcup_{0 \leq t \leq T} B_{R-\omega t}\left(x_{0}\right) \times\{t\} .
$$

The relation between $\omega, R, T$ and $K$ is illustrated in Figure 1 .

Because of Lemma 2.6 and Theorem 2.9, Lemma 2.10 holds for $\tilde{u}:=u_{h}$. This means that

$$
\begin{aligned}
\int_{\mathbb{R}^{N} \times \mathbb{R}^{+}}\left[\left|u_{h}-u\right|\left(\frac{T-t}{T} \rho^{\prime} \omega-\frac{1}{T} \rho\right)\right. \\
\left.+\left(F\left(., \max \left\{u_{h}, u\right\}\right)-F\left(., \min \left\{u_{h}, u\right\}\right)\right) \frac{T-t}{T} \rho^{\prime} \frac{x-x_{0}}{\left|x-x_{0}\right|}\right] d x d t \\
\geq-a_{0} \mu_{h}^{0}(\{\psi(., 0) \neq 0\})-2 \sqrt{b c} \mu_{h}(\{\psi \neq 0\})^{\frac{1}{2}}-a \mu_{h}(\{\psi \neq 0\}) .
\end{aligned}
$$

Now the terms containing $F$ can be estimated as follows:

$$
\begin{gathered}
F\left(., \max \left\{u_{h}, u\right\}\right)-F\left(., \min \left\{u_{h}, u\right\}\right) \frac{T-t}{T} \rho^{\prime} \frac{x-x_{0}}{\left|x-x_{0}\right|} \\
\leq c_{0}\left(M_{0}\right)\left|\max \left\{u_{h}, u\right\}-\min \left\{u_{h}, u\right\}\right| \frac{T-t}{T}\left|\rho^{\prime}\right| \\
\leq c_{0}\left(M_{0}\right)\left|u_{h}-u\right| \frac{T-t}{T}\left|\rho^{\prime}\right|=\omega\left|u_{h}-u\right| \frac{T-t}{T}\left|\rho^{\prime}\right| .
\end{gathered}
$$

Therefore we obtain from (34)

$$
\begin{aligned}
\frac{1}{T} \int_{K}\left|u-u_{h}\right| & =\frac{1}{T} \int_{K} \rho\left(\left|x-x_{0}\right|+\omega t\right)\left|u-u_{h}\right| \leq \frac{1}{T} \int_{\mathbb{R}^{N} \times \mathbb{R}^{+}} \rho\left|u-u_{h}\right| \\
& \leq a_{0} \mu_{h}^{0}(\{\psi(., 0) \neq 0\})+2 \sqrt{b c} \mu_{h}(\{\psi \neq 0\})^{\frac{1}{2}}+a \mu_{h}(\{\psi \neq 0\}),
\end{aligned}
$$

since $(x, t) \in K$ implies that $(x, t) \in B_{R-\omega t}\left(x_{0}\right) \times\{t\}$ and $\rho\left(\left|x-x_{0}\right|+\omega t\right)=1$. 
Now the theorem follows from the following two lemmata.

Lemma 2.12. Let $\psi$ be defined as in (25). Then

$$
\mu_{h}^{0}(\{\psi(.0) \neq 0\}) \leq \int_{\left|x-x_{0}\right|<R+1}\left|u_{0}(x)-u_{h}(x, 0)\right| d x .
$$

Proof. We have

$$
\mu_{h}^{0}(\{\psi(., 0) \neq 0\}) \leq \mu_{h}^{0}\left(\left\{\left|x-x_{0}\right|+\omega t<R+\left.1\right|_{t=0}\right\}\right)
$$

and

$$
\mu_{h}^{0}\left(\left\{\left|x-x_{0}\right|<R+1\right\}\right)=\int_{\left|x-x_{0}\right|<R+1}\left|u_{0}(x)-u_{h}(x, 0)\right| d x .
$$

Lemma 2.13. Let $\psi$ be defined as in (25). Then

$$
\begin{aligned}
\mu_{h}(\{\psi \neq 0\}) \leq & \sum_{n \in I_{0}} \sum_{j \in M\left(t^{n}\right)}\left|u_{j}^{n+1}-u_{j}^{n}\right| \Delta t^{n} h_{j}^{N} \\
& +2 \sum_{n} \sum_{\text {edges }} \Delta t^{n}\left(\Delta t^{n}+h_{j l}\right) \max _{u_{l}^{n} \leq c \leq d \leq u_{j}^{n}}\left(g_{j l}^{n}(d, c)-g_{j l}^{n}(d, d)\right) \delta_{j l}^{n} \\
& +2 \sum_{n} \sum_{\text {edges }} \Delta t^{n}\left(\Delta t^{n}+h_{j l}\right) \max _{u_{l}^{n} \leq c \leq d \leq u_{j}^{n}}\left(g_{j l}^{n}(d, c)-g_{j l}^{n}(c, c)\right) \delta_{j l}^{n} \\
& +2 M_{1} \sum_{n} \sum_{\text {edges }}\left(\Delta t^{n}+h_{j l}\right)^{2} \Delta t^{n} h_{j l}^{N-1} \delta_{j l}^{n},
\end{aligned}
$$

$$
\begin{aligned}
\lambda_{h}(\{\psi \neq 0\}) & \leq \lambda_{h}\left(D_{R+1}\right)=\sum_{n \in I_{0}} \sum_{j \in M\left(t^{n}\right)}\left|u_{j}^{n+1}-u_{j}^{n}\right| \int_{t^{n}}^{t^{n+1}} \int_{T_{j}} \chi_{D_{R+1}} d x d t \\
& \leq \sum_{n \in I_{0}} \sum_{j \in M\left(t^{n}\right)} \Delta t^{n} h_{j}^{N}\left|u_{j}^{n+1}-u_{j}^{n}\right| .
\end{aligned}
$$

This proves the estimate concerning $\lambda_{h}$. Now we have to estimate $\mu_{j l}^{n}$, which is also part of $\mu_{h}$. This measure is defined as (see Definition 2.7)

$$
\begin{aligned}
\left\langle\mu_{j l}^{n}, \psi\right\rangle:=\frac{1}{\left(\Delta t^{n}\right)^{2}\left|T_{j}\right|\left|S_{j l}\right|} \int_{t^{n}}^{t^{n+1}} & \int_{T_{j}} \int_{t^{n}}^{t^{n+1}} \int_{S_{j l}} \int_{0}^{1}\left(h_{j l}+\Delta t^{n}\right) \\
& \times \psi(\gamma+\theta(x-\gamma), s+\theta(t-s)) d \theta d x d t d \gamma d s .
\end{aligned}
$$


First we consider the case when $T_{j} \times\left[t^{n}, t^{n+1}\right] \cap D_{R+1}=\emptyset$ and $T_{l} \times\left[t^{n}, t^{n+1}\right] \cap$ $D_{R+1}=\emptyset$. Since $(\gamma, s) \in T_{j} \times\left[t^{n}, t^{n+1}\left[\right.\right.$ and $(x, t) \in S_{j l} \times\left[t^{n}, t^{n+1}[\right.$, for the convex combination $(\gamma+\theta(x-\gamma), s+\theta(t-s))$ we get

$$
(\gamma+\theta(x-\gamma), s+\theta(t-s)) \in T_{j} \times\left[t^{n}, t^{n+1}[,\right.
$$

and therefore

$$
\psi(\gamma+\theta(x-\gamma), s+\theta(t-s))=0
$$

Otherwise

$$
|\psi(\gamma+\theta(x-\gamma), s+\theta(t-s))| \leq 1
$$

Hence, using the definition of $\delta_{j l}^{n}$ in (32), we obtain

$$
\left\langle\mu_{j l}^{n}, \psi\right\rangle \leq \delta_{j l}^{n}\left(\Delta t^{n}+h_{j l}\right) .
$$

Finally consider the definition of $\nu_{j l}$ in Definition 2.7. As before, we get

$$
\left\langle\nu_{j l}^{n}, \psi\right\rangle \leq \delta_{j l}^{n}\left(\Delta t^{n}+h_{j l}\right)^{2} \Delta t^{n} h_{j l}^{N-1} .
$$

Using the estimates (41), (42) and (43) in the definition of $\mu_{h}$ in (19), we obtain the statement of the lemma.

Now the proof of Theorem 2.11 follows if we use (37), (40) in (36).

Corollary 2.14. Under the assumption of Theorem 2.11, and if $F(x, t, v)=F(v)$ (see Remark 2.4), we have

$$
\int_{K}\left|u-u_{h}\right| \leq T\left(a_{0} \int_{\left|x-x_{0}\right|<R+1}\left|u_{0}(x)-u_{h}(x, 0)\right| d x+a Q+2 \sqrt{b c Q}\right)
$$

where

$$
\begin{aligned}
Q:= & \sum_{n \in I_{0}} \sum_{j \in M\left(t^{n}\right)} \Delta t^{n} h_{j}^{N}\left|u_{j}^{n+1}-u_{j}^{n}\right| \\
& \left.+4 L \sum_{n} \sum_{E\left(t_{n}\right)} \Delta t^{n}\left(\Delta t^{n}+h_{j l}\right) h_{j l}^{N-1}\left|u_{j}^{n}-u_{l}^{n}\right|\right)
\end{aligned}
$$

and $E\left(t_{n}\right)$ is the set of all edges which lie in $M\left(t^{n}\right)$.

Remark 2.15. In the situation of this corollary we have $\|u\|_{B V} \leq\left\|u_{0}\right\|_{B V}$, so that the constant $c$, as defined in (27), can be calculated a priori. In the general case $F=$ $F(x, t, v)$ there exist, at least in two space dimensions, constants $C, C^{\prime}$ depending only on $F, u_{0}, \Omega, T$ and $h / \Delta t^{n}$, such that for all compact sets $\Omega \subset \mathbb{R}^{2}$

$$
\|u\|_{B V(\Omega \times[0, T[)} \leq T e^{C T} C^{\prime} .
$$

See Lemma 7 of [1] for this result. 


\section{NumERICAL TESTS}

In this section we want to confirm the theoretical results of Section 2 by some numerical tests in two space dimensions. Therefore we first introduce an adaptive algorithm, which is based on the a posteriori error estimate of Corollary 2.14. In this adaptive algorithm we can prescribe an error tolerance for the $L^{1}$ - error between the exact and the numerical solution of the test problem. Due to the theoretical results of Corollary 2.14 this prescribed error tolerance should be an upper bound for the actual $L^{1}$-error, which can be computed in this test examples, where the exact solutions are known. In the following subsections this behaviour is analyzed for two test problems.

3.1. An adaptive strategy. For the definition of an adaptive algorithm let us for simplicity look at the scalar conservation law (2) in the situation, where $F(x, t, v)=$ $F(v)$. Due to the estimate of Corollary 2.14 let us first define the following local estimators:

$$
\begin{aligned}
\left(\eta_{0}\right)_{j} & =\int_{T_{j}}\left|u_{0}(x)-u_{h}(x, 0)\right| d x \\
\left(\eta_{t}\right)_{j}^{n} & =h_{j}^{2}\left|u_{j}^{n+1}-u_{j}^{n}\right| \\
\left(\eta_{x}\right)_{j l}^{n} & =\left(\triangle t^{n}+h_{j l}\right) h_{j l}\left|u_{j}^{n}-u_{l}^{n}\right| .
\end{aligned}
$$

Additionally we define the following upper bounds for $\alpha, \beta \in(0,1)$ :

$$
\begin{aligned}
B_{0} & =\frac{\operatorname{Tol}_{0}}{a_{0} T \bar{M}^{0}}, \\
B_{t} & =\min \left\{\alpha \frac{\mathrm{Tol}_{t}}{a T^{2} \bar{M}^{n}},(1-\alpha)^{2} \frac{\mathrm{Tol}_{t}^{2}}{4 b c T^{3} \bar{M}^{n}}\right\} \\
B_{x} & =\min \left\{\beta \frac{\mathrm{Tol}_{x}}{4 L a T^{2} \bar{E}^{n}},(1-\beta)^{2} \frac{\mathrm{Tol}_{x}^{2}}{16 L b c T^{3} \bar{E}^{n}}\right\}
\end{aligned}
$$

Here $a_{0}, a, b, c, T$ and $L$ are the constants of Corollary 2.14. $\bar{M}^{n}, \bar{E}^{n}$ denote the number of triangles and edges in $M\left(t^{n}\right), E\left(t^{n}\right)$ respectively, and $\mathrm{Tol}_{0}, \mathrm{Tol}_{t}, \mathrm{Tol}_{x}$ are the tolerances for the estimators $\eta_{0}, \eta_{t}$ and $\eta_{x}$. Moreover let $\mathrm{Tol}_{0}, \mathrm{Tol}_{t}$, Tol $x$ be chosen in such a way that for a prescribed tolerance Tol we have

$$
\mathrm{Tol}=\mathrm{Tol}_{0}+\mathrm{Tol}_{t}+\mathrm{Tol}_{x}
$$

With this definitions we can now state the following corollary.

Corollary 3.1. Let the assumptions of Corollary 2.14 be fulfilled. If in addition $\left(\eta_{0}\right)_{j} \leq B_{0},\left(\eta_{t}\right)_{j}^{n} \leq B_{t}$ and $\left(\eta_{x}\right)_{j l}^{n} \leq B_{x}$ for all $n \in I_{0}, j \in M\left(t^{n}\right)$ and all $j, l$, such that $S_{j l}$ is an edge of $E\left(t^{n}\right)$, then the following estimate holds for any prescribed tolerance Tol :

$$
\int_{K}\left|u-u_{h}\right| \leq \text { Tol }
$$


Proof. Using the assumptions of the corollary, we get, by Corollary 2.14,

$$
\begin{aligned}
& \int_{K}\left|u-u_{h}\right| \leq T a_{0} \int_{\left|x-x_{0}\right|<R+1}\left|u_{0}(x)-u_{h}(x, 0)\right| d x \\
&+a\left(\sum_{n \in I_{0}} \sum_{j \in M\left(t^{n}\right)} \Delta t^{n} h_{j}^{2}\left|u_{j}^{n+1}-u_{j}^{n}\right|\right. \\
&\left.\left.+4 L \sum_{n \in I_{0}} \sum_{S_{j l} \in E\left(t^{n}\right)} \Delta t^{n}\left(\Delta t^{n}+h_{j l}\right) h_{j l}\left|u_{j}^{n}-u_{l}^{n}\right|\right)\right) \\
&+2 \sqrt{b c}\left(\sum_{n \in I_{0}} \sum_{j \in M\left(t^{n}\right)} \Delta t^{n} h_{j}^{2}\left|u_{j}^{n+1}-u_{j}^{n}\right|\right. \\
&\left.\left.+4 L \sum_{n \in I_{0}} \sum_{S_{j l} \in E\left(t^{n}\right)} \Delta t^{n}\left(\Delta t^{n}+h_{j l}\right) h_{j l}\right)^{1 / 2}\left|u_{j}^{n}-u_{l}^{n}\right|\right) \\
& \leq T\left(a_{0} \sum_{j \in M(0)} B_{0}+a \sum_{n \in I_{0}} \sum_{j \in M\left(t^{n}\right)} \Delta t^{n} B_{t}+4 L a \sum_{n \in I_{0}} \sum_{S_{j l} \in E\left(t^{n}\right)} \Delta t^{n} B_{x}\right. \\
&\left.\quad+2 \sqrt{b c} \sqrt{\sum_{n \in I_{0}} \sum_{j \in M\left(t^{n}\right)} \Delta t^{n} B_{t}+4 L \sum_{n \in I_{0}} \sum_{S_{j l} \in E\left(t^{n}\right)} \Delta t^{n} B_{x}}\right) \\
& \leq \operatorname{Tol}_{0}+\alpha \operatorname{Tol}_{t}+\beta \operatorname{Tol}_{x}+\sqrt{(1-\alpha)^{2} \operatorname{Tol}_{t}^{2}+(1-\beta)^{2} \operatorname{Tol}_{x}^{2}} \\
& \leq \operatorname{Tol} \quad
\end{aligned}
$$

Due to this result the adaptive algorithm reads as follows:

for all $n \in I_{0}\{$

for all $j \in M\left(t^{n}\right)$ and all $S_{j l} \in E\left(t^{n}\right)\{$

coarse $T_{j}$ and/or $T_{l}$ if $\left(\eta_{0}\right)_{j} \leq \theta B_{0},\left(\eta_{t}\right)_{j}^{n} \leq \theta B_{t}$ or $\left(\eta_{x}\right)_{j l}^{n} \leq \theta B_{x}$; refine $T_{j}$ and/or $T_{l}$, until $\left(\eta_{0}\right)_{j} \leq B_{0},\left(\eta_{t}\right)_{j}^{n} \leq B_{t}$ and $\left(\eta_{x}\right)_{j l}^{n} \leq B_{x}$;

\} calculate the next time step on the resulting mesh;

\}

Here $\theta$ is a threshold value, which should be chosen in $(0,1)$ depending on the refinement rules of the mesh.

Remark 3.2. Let us remark that the conditions $\left(\eta_{0}\right)_{j} \leq B_{0},\left(\eta_{t}\right)_{j}^{n} \leq B_{t}$ and $\left(\eta_{x}\right)_{j l}^{n} \leq$ $B_{x}$ can always be fulfilled, as the estimators converge faster towards zero than the upper bounds do, when $h_{j}, h_{j l}$ converge to zero.

Furthermore, it is important to state that the time interval $\triangle t^{n}$ is also adapted in this algorithm, because $\Delta t^{n}$ is directly connected to $h_{\min }$ through the CFL condition.

In the following two subsections we now want to verify the statement of Corollary 3.1 for this adaptive algorithm in two test situations, where the exact solution of the problem is known. Therefore we prescribe an error tolerance Tol and compare the actual error $\int_{K}\left|u-u_{h}\right|$ to this tolerance. 
3.2. A Burgers type problem. Let us consider the nonlinear conservation law

(2) together with the initial condition (3) in $\mathbb{R}^{2}$, where

$$
F(x, t, u)=\left(\begin{array}{l}
u^{2} \\
u^{2}
\end{array}\right) \quad \text { and } \quad u_{0}(x)=\left\{\begin{array}{lll}
2, & \text { if } & \frac{x_{1}+x_{2}}{2}-0.5 \leq 0, \\
1, & \text { if } & \frac{x_{1}+x_{2}}{2}-0.5>0 .
\end{array}\right.
$$

Then the exact solution of this Burgers type problem is

$$
u(x, t)=\left\{\begin{array}{lll}
2, & \text { if } & \frac{x_{1}+x_{2}}{2}-0.5 \leq 3 t \\
1, & \text { if } & \frac{x_{1}+x_{2}}{2}-0.5>3 t .
\end{array}\right.
$$
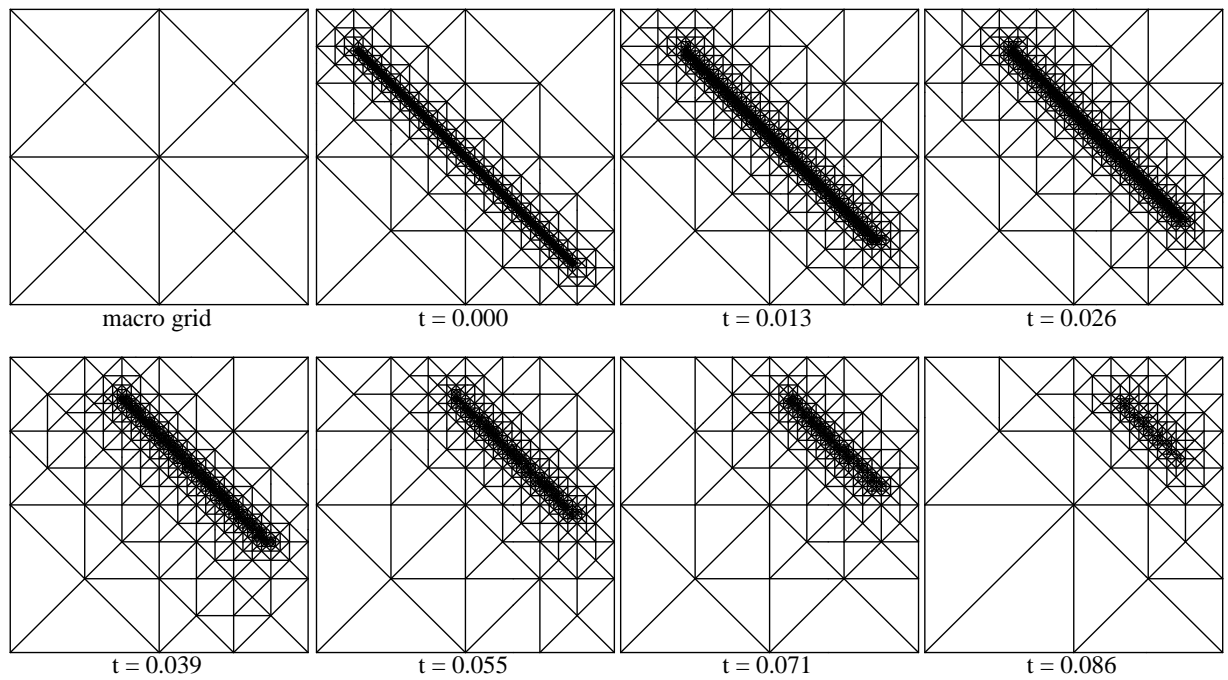

Figure 2. Macro grid and adaptive grids at different time steps for the Burgers type problem.
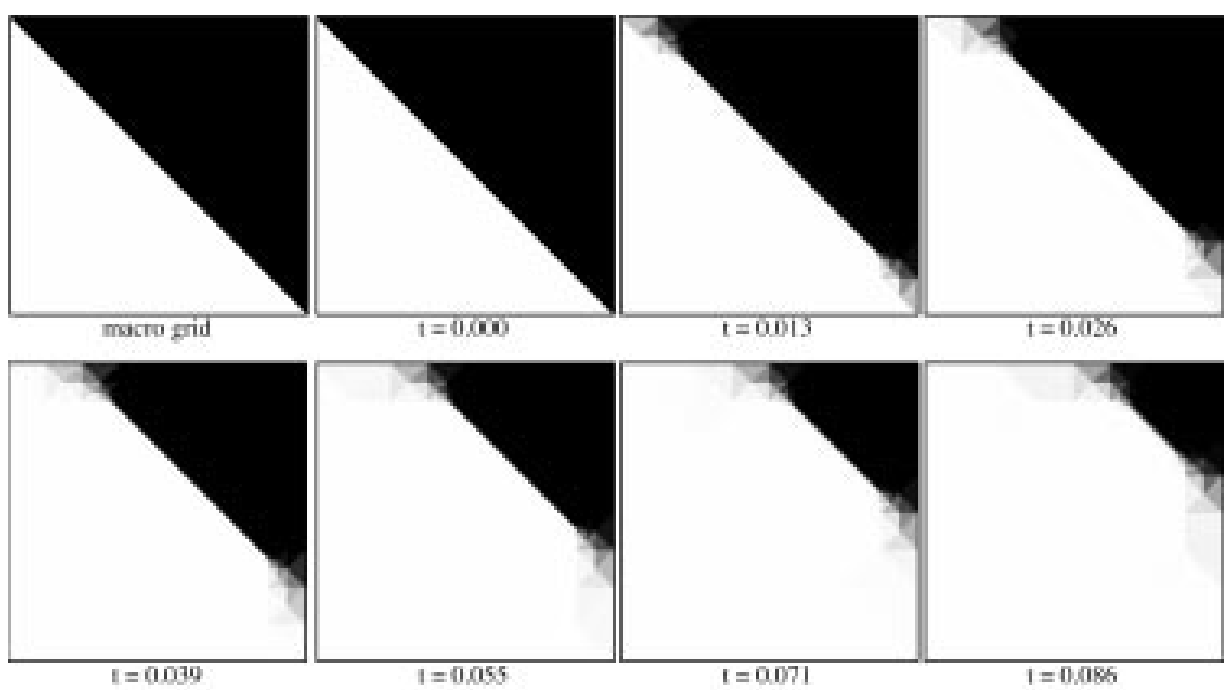

Figure 3. The traveling shock of the Burgers type problem. 
TABLE 1. Comparison between the prescribed tolerance and the actual error for the Burgers type problem.

\begin{tabular}{|c||c|c|c|c|c|c|c|}
\hline TOL & 0.024 & 0.018 & 0.012 & 0.008 & 0.004 & 0.002 & 0.001 \\
\hline \hline$\left\|u-u_{h}\right\|_{1}$ & 0.01989 & 0.00975 & 0.00937 & 0.00526 & 0.00147 & 0.00052 & 0.00016 \\
\hline
\end{tabular}

In Figure 2 the adaptive refined grids for different time steps are shown. In this example the error should be controlled in the set $B_{0.1}(0.75,0.75)$. Theorem 2.11 indicates that we have to control the error in the whole cone of dependence. This means we can choose $K$ as the cone of dependence corresponding to $B_{0.1}(0.75,0.75)$. Due to the choice of the cone $K$, where the error should be controlled, the grid is only refined inside the cone. The figure shows how the dependence region concentrates to a small disc $B_{0.1}(0.75,0.75)$ as $t$ reaches $T=0.1$. Since the last time steps contribute less to the $L^{1}$-error over $K$ than the first time steps do, the grid is not that fine at the end of the calculations. The corresponding shock solution is shown in Figure 3. Here the grey values indicate the values of the solution $u_{h}$.

Table 1 shows the prescribed tolerances for different simulations of the Burgers type problem and the corresponding actual errors between the exact solution and the discrete solution of the finite volume scheme.

3.3. The rotating cylinder problem. Let us consider the linear conservation law (2) together with the initial condition $(3)$ in $\mathbb{R}^{2}$, where

$$
F(x, t, u)=\left(\begin{array}{rr}
-x_{2} & u \\
x_{1} & u
\end{array}\right) \quad \text { and } \quad u_{0}(x)=\left\{\begin{array}{ll}
1, & \text { if } \\
0, & \text { else. }
\end{array}\left|x-(1,0)^{\top}\right|<0.5,\right.
$$

Then the exact solution of this rotating cylinder problem is

$$
u(x, t)=u_{0}\left(\left(\cos (t) x_{1}+\sin (t) x_{2},-\sin (t) x_{1}+\cos (t) x_{2}\right)^{\top}\right) .
$$

The numerical solution of the rotating cylinder problem with the adaptive algorithm is shown in Figure 5, whereas Figure 4 shows the underlying adaptive grid. The set $K$ is chosen such that $K \cap \Omega \times T=B_{0.6}(-1.0,0.0)$, where $T=3.15$. Table 2 shows the prescribed tolerances for different simulations of the rotating cylinder problem and the corresponding actual errors between the exact solution and the discrete solution of the finite volume scheme.

TABle 2. Comparison between the prescribed tolerance and the actual error for the rotating cylinder problem.

\begin{tabular}{|c||c|c|c|c|c|c|c|}
\hline TOL & 3.0 & 2.0 & 1.6 & 1.2 & 0.8 & 0.4 & 0.2 \\
\hline \hline$\left\|u-u_{h}\right\|_{1}$ & 2.28430 & 1.54125 & 1.12592 & 0.878921 & 0.61415 & 0.33538 & 0.17748 \\
\hline
\end{tabular}





Figure 4. Macro grid and adaptive grids at different time steps for the rotating cylinder problem.
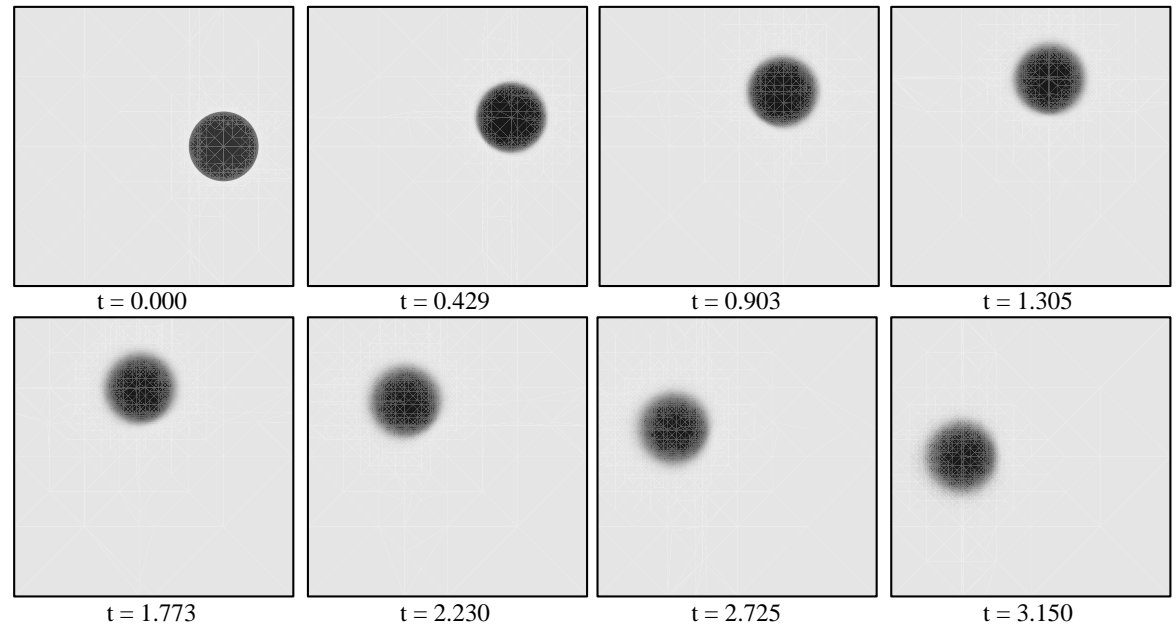

Figure 5. The numerical solution of the rotating cylinder problem.

\section{ACKNOWLEDGMENT}

We would like to thank the referees for suggesting that we should localize condition (6) in [1] and use the weaker condition (9) instead.

\section{REFERENCES}

[1] C. Chainais-Hillairet: Finite volume schemes for a nonlinear hyperbolic equation. Convergence towards the entropy solution and error estimates. Preprint http://umpa.enslyon.fr/UMPA/Prepublications/0205/, ENS Lyon (1997).

[2] Cockburn,B. Gremaud, P.A.: An error estimate for finite element methods for scalar conservation laws. IMA Preprint Series No.1144 (1993).

[3] Cockburn,B. Gremaud, P.A.: Error estimates for finite element methods for scalar conservation laws. SIAM J. Numer. Anal., 33 (1996), 522-554. MR 97e:65096 
[4] Cockburn,B., Coquel,F., LeFloch, P.: An error estimate for finite volume methods for multidimensional conservation laws. Math. Comput., 63 (1994), 77-103. MR 95d:65078

[5] Cockburn,B., Coquel,F., LeFloch, P.: An error estimate for high-order accurate finite volume methods for scalar conservation laws. Math. Comput. to appear.

[6] Dörfler, W.: A convergent adaptive algorithm for Poisson's equation. SIAM J. Numer. Anal., 33 (1996), 1106-1124. MR 97e:65139

[7] Eriksson, K., Johnson,C.: Adaptive finite element methods for parabolic problems I: A linear model problem. SIAM J. Numer. Anal., 28 (1991), 43-77. MR 91m:65274

[8] Eriksson, K., Johnson,C.: Adaptive finite element methods for parabolic problems IV: Nonlinear Problems. SIAM J. Numer. Anal., 32 (1995), 1729-1749. MR 96i:65081

[9] Houston, P., Süli, E.,: Adaptive Lagrange-Galerkin methods for unsteady convectiondominated diffusion problems. Oxford University Computing Laboratory, Numerical Analysis Group, Report Nr. 95/24.

[10] Johnson,C., Szepessy, A.: Adaptive finite element methods for conservation laws based on a posteriori error estimates. Commun. Pure Appl. Math., 48 (1995), 199-234. MR 97b:76084

[11] Kröner, D., Noelle, S., Rokyta, M.: Convergence of higher order upwind finite volume schemes on unstructured grids for scalar conservation laws in several space dimensions. Numer. Math., 71 (1995), 527-560. MR 97j:65087

[12] Kröner, D., Rokyta, M.: Convergence of upwind finite volume schemes for scalar conservation laws in two dimensions. SIAM J. Numer. Anal., 31 (1994), 324-343. MR 95e:65085

[13] Kröner, D., Rokyta, M.: A priori error estimates for upwind finite volume schemes in several space dimensions. Preprint 37, Freiburg, Mathematische Fakultät (1996). To appear in SIAM J. Numer. Anal..

[14] Mackenzie, J.A., Süli, E., Warnecke, G.: A posteriori analysis for Petrov-Galerkin approximations of Friedrichs systems. Oxford University Computing Laboratory, Numerical Analysis Group, Report Nr. 95/01.

[15] Noelle, S.: Convergence of higher order finite volume schemes on irregular grids. Adv.Comput.Math., 3 (1995), 197-218. MR 96a:65137

[16] Süli, E., Houston, P.: Finite element methods for hyperbolic problems: aposteriori analysis and adaptivity. In: Duff, I. S. (ed.) et al., The state of the art in numerical analysis, Clarendon Press, Oxford (1997), 441-471. CMP 98:14

[17] Tadmor, E.: Local error estimates for discontinuous solutions of nonlinear hyperbolic equations. SIAM J. Numer. Anal., 28 (1991), 891-906. MR 92d:35190

[18] Tveito, A., Winther, R.: On the rate of convergence to equilibrium for a system of conservation laws with a relaxation term. SIAM J. Math. Anal., 28 (1997), 136-161. MR 98a:35087

[19] Verfürth, R.: Robust a posteriori error estimators for the singularly perturbed Helmholtz equation. Fakultät für Mathematik, Bochum, Bericht Nr. 201 (1996).

[20] Vila, J.P.: Convergence and error estimates in finite volume schemes for general multidimensional scalar conservation laws. I Explicit monotone schemes. RAIRO, Modélisation Math. Anal. Numér., 28 (1994), 267-295. MR 96d:65150

Institut für Angewandte Mathematik, Universität Freiburg, Herrmann-Herder-Str. 10, 79104 Freiburg, Germany

E-mail address: dietmar@mathematik.uni-freiburg.de

E-mail address: mario@mathematik.uni-freiburg.de 